\title{
Preventive analgesia with pregabalin in laparoscopic cholecystectomy post-operated patients
}

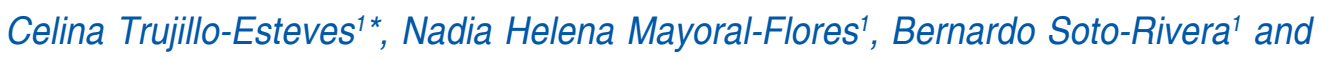 \\ José Vicente Rosas-Barrientos ${ }^{2}$ \\ ${ }^{1}$ Anesthesiology Department; ${ }^{2}$ Research Department. Hospital Regional 10. de Octubre, Instituto de Seguridad y Servicios Sociales de los \\ Trabajadores del Estado, City of Mexico, Mexico
}

\begin{abstract}
Background: Preventive analgesia is the administration of an analgesic drug with the aim of attenuating post-operative pain, hyperalgesia and allodynia. Its use is justified in order to offer analgesia and reduce anxiety in patients undergoing laparoscopic procedures. Objective: To evaluate if pregabalin in a dose of $1 \mathrm{mg} / \mathrm{kg}$ of weight is effective as preventive analgesia in post-operated laparoscopic cholecystectomy patients. Methods: A single-blind controlled clinical trial was conducted, which included 60 patients scheduled for laparoscopic cholecystectomy randomly divided into 2 groups, where Group 1 received placebo and Group 2 received pregabalin a daily dose $72 \mathrm{~h}$ prior to surgical intervention. The intensity of pain was assessed using the numeric analog scale at 1, 2, 6, 12 and 24 post-operative $h$, as well as the level of presurgical anxiety with the Hamilton scale. Results: Pain reduction was demonstrated in patients in the pregabalin group from the 1st $h(p=0.002)$, later the decrease in pain was more noticeable compared to patients who were given placebo $(p<0.001)$, the same happened with the anxiety level evaluated with the Hamilton scale $(p<0.005)$. Conclusion: The use of pregabalin as preventive analgesia turns out to be effective in the post-operative period and the pre-operative anxiety with minimal adverse effects in the post-operated patients of laparoscopic cholecystectomy.
\end{abstract}

KEY WORDS: Preventive analgesia. Postoperative pain. Pregabalin. Laparoscopic cholecystectomy.

\section{Introduction}

Preventive analgesia consists of administration of drugs to achieve an analgesic state prior to surgical trauma in order to decrease peripheral and central sensitive response to pain, by means of which interrupting the inflammation-pain-hyperalgesia circle and increased painful stimulus is attempted ${ }^{1}$. Pregabalin is a neuromodulator used in the treatment of neuropathic pain, and several works indicate that it can be administered for the prevention of postoperative pain ${ }^{2}$. Postoperative pain control in patients undergoing laparoscopic cholecystectomy is not only a challenge for the anesthesiologist due to the type of pain experienced by patients, but also a commitment to them ${ }^{3,4}$. When considering postoperative pain resulting from surgical manipulation, moderate to severe in intensity, using combinations of non-steroidal anti-inflammatory drugs, opiates and neuromodulators is required, and it is justified with the purpose to offer an adequate analgesia and reduce anxiety in patients undergoing laparoscopic cholecystectomy 5 . The purpose of the present study is to assess whether pregabalin is effective when used as preventive analgesia for postsurgical pain in patients undergoing laparoscopic cholecystectomy.

\section{Method}

A controlled, blinded, balanced-block randomized clinical trial was carried out in a sample of 60 patients. According to sample size calculation by proportion estimation, 26 patients were required per group, and
Gac Med Mex. 2019;155 (Suppl 1):S13-S16

Contents available at PubMed www.gacetamedicademexico.com 
it was adjusted to 30 patients for possible losses. Patients of either gender, aged between 18 and 60 years, with American Society of Anesthesiologists (ASA) physical status classification class 1 and 2, selected among the surgical population programmed for laparoscopic cholecystectomy, were included after granting informed consent. The study was carried out under the principles of bioethics and was previously approved by the Committees of Research and Ethics at the Regional Hospital $1^{\circ}$ de Octubre of the Institute of Social Security and Services of State Workers, with institutional registry number RPI 211.2012.

During pre-anesthetic assessment, if the patient met the inclusion criteria, he/she was invited to participate in the study and the informed consent process was carried out. Patients were distributed in two groups; the randomization procedure was by blocks, using a table of random numbers.

Both groups received capsules with similar physical characteristics, with contents adjusted close to patient weight; thus, the treatment group received pregabalin $1 \mathrm{mg} / \mathrm{kg}$ of weight and the placebo group received capsules with the excipient. Patients were instructed to take one capsule every 24 hours during the three days prior to surgery.

Prior to the anesthetic procedure, the Hamilton-Hunt Scale was applied to measure preoperative levels of anxiety. In addition, any intentionally-sought adverse event referred by the patients, such as dizziness, somnolence, blurred vision or lower limb edema, was also recorded.

The anesthetic procedure was intended to be similar for all patients, if their physical status and background allowed; thus, anesthetic induction was carried out with propofol $2 \mathrm{mg} / \mathrm{kg}$, basal narcosis with fentanyl $3 \mathrm{mcg} / \mathrm{kg}$ and the neuromuscular relaxant used was cisatracurium at a dose of $100 \mathrm{mcg} / \mathrm{kg}$; anesthetic maintenance was carried out with sevoflurane at a dose of 1-1.2 Minimum Alveolar Concentration (MAC) and, finally, the dose of analgesic opioid fentanyl was $5 \mathrm{mcg} / \mathrm{kg} /$ hour. Vital signs, such as heart rate, non-invasive blood pressure and exhaled $\mathrm{CO}_{2}$ every 5 minutes were recorded. Once the surgical procedure concluded, the patients were transferred to the post-anesthetic recovery unit, where pain intensity was assessed.

The first pain evaluation with the numeric analog scale (NAS) (0 no pain-10 severe pain) was carried out in all patients upon arrival to the recovery unit, at 60 minutes and at 2, 6, 12 and 24 post-surgery hours. Vital signs such as non-invasive blood pressure, heart rate and respiratory rate were also recorded.
Table 1. Patient baseline characteristics according to study group

\begin{tabular}{|l|c|c|c|}
\hline Variable & Pregabalin group & Placebo group & $p^{*}$ \\
\hline Age, years & $43.3 \pm 12.9$ & $46.9 \pm 8.5$ & 0.198 \\
\hline $\begin{array}{l}\text { Gender, } \mathrm{n}(\%) \\
\text { Males } \\
\text { Females }\end{array}$ & $2(7)$ & $7(23)$ & 0.15 \\
\hline Weight (kg) & $28(93)$ & $23(77)$ & \\
\hline SBP (mmHg) & $13.7 \pm 14.6$ & $73.4 \pm 15.6$ & 0.665 \\
\hline DBP (mmHg) & $79.8 \pm 9.4$ & $78.0 \pm 10.5$ & 0.502 \\
\hline HR (bpm) & $81.2 \pm 13.4$ & $81.8 \pm 8.7$ & 0.838 \\
\hline RR (bpm) & $16.7 \pm 2.2$ & $16.2 \pm 2.5$ & 0.442 \\
\hline $\begin{array}{l}\text { Hamilton previous } \\
72 \mathrm{~h}\end{array}$ & $13.7 \pm 5.7$ & $11.2 \pm 4.9$ & 0.077 \\
\hline
\end{tabular}

*Data for quantitative variables expressed as the mean and standard deviation. Comparison test for quantitative Student's t-test and qualitative chi-square test. SBP: systolic blood pressure; DBP: diastolic blood pressure; HR: heart rate; RR: respiratory rate.

Postoperative pain control was carried out with the administration of ketorolac $1 \mathrm{mg} / \mathrm{kg}$ of weight and tramadol $1 \mathrm{mg} / \mathrm{kg}$ intravenously every 8 hours, with the number of analgesic rescues being recorded.

Our independent variable was pretreatment with pregabalin and the dependent variable postsurgical pain.

\section{Statistical analysis}

Frequency and central tendency measures were used. For inferential statistics, Student's t-test was used for quantitative variables, and the non-parametric Mann-Whitney U-test was used for independent samples, and for qualitative variables, the chi-square test. Statistical significance was set at a p-value $<0.05$.

\section{Results}

Sixty patients undergoing laparoscopic cholecystectomy were included and randomly assigned to receive pregabalin $1 \mathrm{mg} / \mathrm{kg}$ of weight by oral route every 24 hours for three days prior to surgery, or starch tablets under the same administration schedule.

The demographic characteristics of the study population were homogeneous and are shown in the Table 1.

Analgesic results were statistically significant $(p=0.001)$ in group 2 pregabalin-treated patients with respect to the placebo-treated group 1 (Fig. 1).

In the assessment of the degree of anxiety, group 1 patients reported less difficulty falling asleep and maintaining sleep $(p<0.006)$ (Fig. 2). 


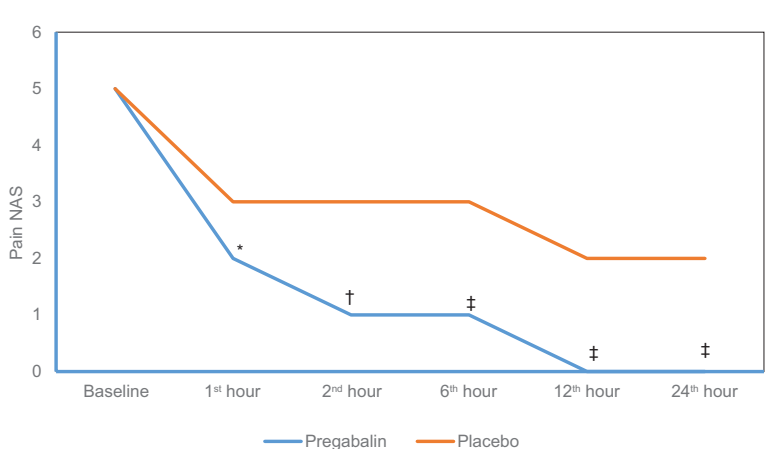

Figure 1. Numerical Analog Scale (NAS) comparison by groups at $1^{\text {st }}$ hour, $2^{\text {nd }}$ hour, $6^{\text {th }}$ hour, $12^{\text {th }}$ hour and at 24 hours.

${ }^{*} p=0.002$

${ }^{t} p=0.001$

${ }^{\neq} p<0.0001$

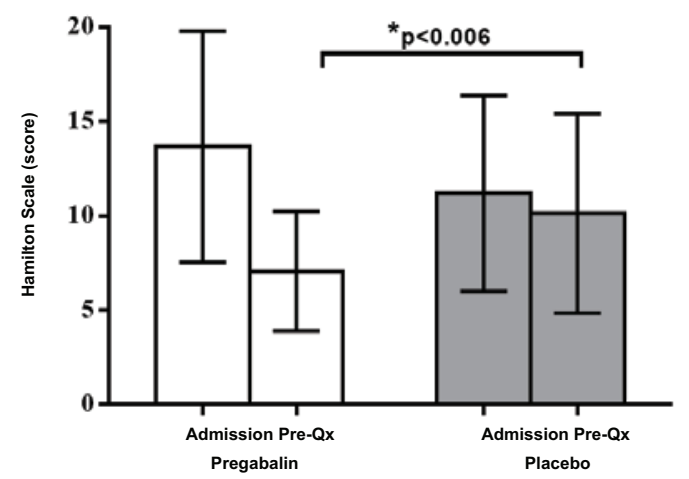

Figure 2. Anxiety score comparison at admission and at pre-surgical moment (Pre-Qx). ${ }^{*} p<0.006$.

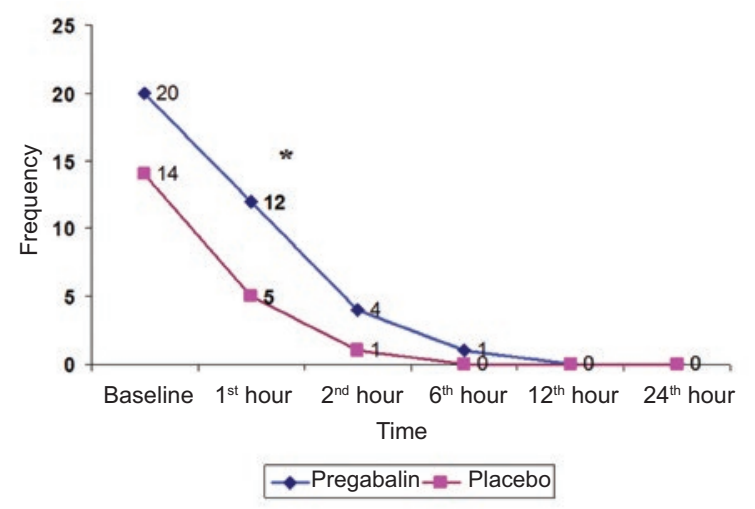

Figure 3. Frequency of dizziness at $1^{\text {st }}$ hour, $2^{\text {nd }}$ hour, $6^{\text {th }}$ hour, $12^{\text {th }}$ hour and at 24 hours ${ }^{*} p<0.05$.

Regarding adverse effects, the most common was dizziness, without reaching statistical significance or forcing treatment interruption in any patient (Fig. 3).

A regards physiological variables, pregabalin at 24 hours post-surgery only caused respiratory rate variation, with statistical significance $(p=0.02)$; even so, it still remained within physiologically normal values $(19.2 \pm 0.57)$.

\section{Discussion}

In laparoscopic cholecystectomy, pain has different origin and installation time, and visceral and parietal pain have preference within the first eight hours. In the subsequent 24 to 48 hours, pain will establish mainly on the right shoulder and main have acute neuropathic pain characteristics. This pain is widely recognized as the cause of post-laparoscopic cholecystectomy pain, and one of the responsible mechanisms is phrenic nerve irritation ${ }^{3,4}$.

Currently, neuropathic-type pain is difficult to control and does not adequately respond to conventional analgesics such as non-steroidal anti-inflammatory drugs and opioid analgesics, in addition to that with the prescription of the latter there is always the risk that the patient experiences postoperative nausea and vomiting, which can complicate post-surgical recovery ${ }^{6}$.

This gives rise to the search for new treatment alternatives to prevent pain and favor the reduction of analgesic opioid doses.

Pregabalin has been used for preventive analgesia, although so far there is no consensus regarding how many days of treatment and at which dose it should be prescribed to be effective in the control of this symptom and produce minimal adverse effects ${ }^{7}$.

Dworkin and Freynhagen ${ }^{8,9}$ report that the dose whereby pregabalin should be started to have an adequate postoperative analgesia is $150 \mathrm{mg} /$ day, with $75 \mathrm{mg}$ administered every 12 hours, a schedule by means of which they obtained moderate to complete postsurgical pain decrease.

In our study, we decided to use an average prescription of 3 days prior to surgery, given that we found different studies that started it from one week to one hour prior to the surgical procedure. We also used the minimum required dose of $1 \mathrm{mg} / \mathrm{kg}$ of weight every 24 hours to look for the analgesic effect in the postoperative period and to determine whether at this dose there was still the presence of the most frequently reported adverse effects with the use of pregabalin, such as dizziness, somnolence, blurred vision and perimaleolar edema.

This is unlike other studies, where doses of up to $300 \mathrm{mg} /$ day were used, but that are more focused on the analgesic effect and on opioid analgesics consumption decrease in the postoperative period, and do not deliberately seek the adverse effects that sometimes make patients have poor adherence or even discontinue treatment, or only look for certain adverse effects such as ataxia or confusion ${ }^{6,10}$. 
Even so, regarding adverse effects, some authors report that only $10 \%$ of their patients experienced them, and that in $15 \%$ of them, adverse effects drove them to discontinue the medication. Other authors claim that pregabalin flexible doses do not decrease the incidence of adverse effects ${ }^{5,8,11}$.

In our study, we accepted the hypothesis that pregabalin minimum dose of $1 \mathrm{mg} / \mathrm{kg} /$ day is effective as preventive analgesia; the adverse effects that occurred were minimal and did not require discontinuing the medication ${ }^{2}$.

Previous works on subjects with pain of similar characteristics also verified that pain intensity decreased with a single dose of pregabalin of $75-150 \mathrm{mg} /$ day, while in our study the dose was, on average, $75 \mathrm{mg} /$ day and was administered 3 days prior to surgery also looking to obtain an anxiolytic effect, with similar results being found in both studies ${ }^{12,13}$.

On the other hand, regarding the anxiety symptom, Argawal et al. report that depression and anxiety are important contributing factors to the onset of pain. Sharon refers that there are multiple factors that contribute to pain, such as personality, previous experience of pain and even genetic factors ${ }^{8}$.

Several studies refer that pregabalin is an effective treatment in patients with moderate to severe generalized anxiety disorders. We assessed pre-surgical anxiety intensity with the Hamilton-Hunt Scale, although we did not find a significant decrease, since our patients mostly had only a mild degree of anxiety, which may be related to pre-surgical diagnosis and to the fact that laparoscopic surgery is not considered a high-risk procedure, which does not cause much anxiety in patients.

Finally, we consider that to understand the variation of results between different authors and our study, it should be considered that it might depend on two factors, mainly:

- In addition to being approved for use as anticonvulsants, the antinociceptive capacity of gabapentinoids was recognized in the decade of 1990 , and later its use was accepted in the treatment of chronic neuropathic pain of peripheral origin, in generalized anxiety disorders and as part of multimodal analgesia in the perioperative period ${ }^{14}$.

- Preventive analgesia is not yet a common practice for the treatment of post-surgical pain, controversies in the evidence about its effectiveness entail for it to be a not-yet viable option for usual practice, and even less if the use of gabapentinoids is considered.
Although the number of published studies assessing the role of pregabalin in the postsurgical pain scenario is very limited, the results found are promising, not only in pain relief in this period, but also for the reduction of anxiety in any type of surgery. There is a coincidence regarding how pregabalin significantly decreases the requirement of opioid analgesics during trans-anesthetic and mainly post-anesthetic periods, as well as in that it also decreases non-steroidal antiinflammatory drugs consumption and reduces postsurgical pain, with few adverse effects ${ }^{10,12,15}$.

Finally, we consider that since pain is a subjective symptom that can be modified by numerous variables, the next step in our research is to look for pain control by comparing different doses and, together with the decrease in opioid analgesics requirements, to objectively know gabapentinoids real analgesic effect in the perioperative period.

\section{References}

1. Sáez VP. Revisión: efecto de la analgesia preventiva en el dolor postoperatorio. Rev Esp Anestesiol Reanim. 2012;59:43-50.

2. Mishra R, Tripathi M, Chandola HJA. Comparative clinical study of gabapentin and pregabalin for postoperative analgesia in laparoscopic cholecystectomy. Anesth Essays Res. 2016;10:201.

3. Gurunathan U, Rapchuk IL, King G, Barnett AG, Fraser JF. The effect of pregabalin and celecoxib on the analgesic requirements after laparoscopic cholecystectomy: a randomized controlled trial. J Anesth. 2016;30:64-71.

4. Baron R, Mayoral V, Leijon G, Binder A, Steigerwald I, Serpell MJ, et al. $5 \%$ lidocaine medicated plaster versus pregabalin in post-herpetic neuralgia and diabetic polyneuropathy: an open-label, non-inferiority two-stage RCT study. Curr Med Res Opin. 2009;25:1663-76.

5. Buvanendran A, Kroin JS, Della Valle CJ, Kari M, Moric M, Tuman K, et al. Perioperative oral pregabalin reduces chronic pain after total knee arthroplasty: a prospective, randomized, controlled trial. Anesth Analg. 2010;110:199-207.

6. Schulmeyer MCC, de la Maza J, Ovalle C, Farias C, Vives I. Analgesic effects of a single preoperative dose of pregabalin after laparoscopic sleeve gastrectomy. Obes Surg. 2010;20:1678-81.

7. Karaca O, Pınar HU, Turk E, Dogan R, Ahiskalioglu A, Solak SK. Effects of single-dose preemptive pregabalin and intravenous ibuprofen on postoperative opioid consumption and acute pain after laparoscopic cholecystectomy. Cholecystectomy J Invest Surg 2017;20:1-7.

8. Agarwal A, Gautam S, Gupta D, Agarwal S, Singh P, Singh UJ. Evaluation of a single preoperative dose of pregabalin for attenuation of postoperative pain after laparoscopic cholecystectomy. Br J Anaesth 2008; 101:700-4.

9. O'Connor AB, Dworkin RH. Treatment of neuropathic pain: an overview of recent guidelines. Am J Med 2009; 122: S22-S32.

10. Balaban F, Yağar S, Özgök A, Koç M, Güllapoğlu HJ. A randomized, placebo-controlled study of pregabalin for postoperative pain intensity after laparoscopic cholecystectomy. J Clin Anesth. 2012;24:175-8.

11. Jokela R, Ahonen J, Tallgren M, Haanpää M, Korttila KJP. A randomized controlled trial of perioperative administration of pregabalin for pain after laparoscopic hysterectomy. Br J Anaesth. 2008 ; 134:106-12.

12. Gupta P, Saxena A, Chaudhary LJA. Effect of pregabalin premedication on the requirement of anesthetic and analgesic drugs in laparoscopic cholecystectomy: Randomized Comparison of Two Doses. Anesth Essays Res. 2017; 11:330-333.

13. Sarakatsianou C, Theodorou E, Georgopoulou S, Stamatiou G, Tzovaras GJ. Effect of pre-emptive pregabalin on pain intensity and postoperative morphine consumption after laparoscopic cholecystectomy. Anesth Essays Res. 2017; 11:330-333.

14. Shimony N, Amit U, Minz B, Grossman R, Dany MA, Gonen L, et al. Perioperative pregabalin for reducing pain, analgesic consumption, and anxiety and enhancing sleep quality in elective neurosurgical patients: a prospective, randomized, double-blind, and controlled clinical study. J Neurosurg. $2016 ; 125: 1513-1522$.

15. Esmat IM, Farag HM. Comparative study between paracetamol and two different doses of pregabalin on postoperative pain in laparoscopic cholecystectomy. Saudi J Anaesth. 2015;9:376-80. 